\title{
FWFusion: Fuzzy Whale Fusion model for MRI multimodal image fusion
}

\author{
HANMANT VENKETRAO PATIL ${ }^{1, *}$ and SURESH D SHIRBAHADURKAR ${ }^{2}$ \\ ${ }^{1}$ Department of Electronics and Telecommunication, NDMVPS's KBT COE, Nashik 422013, India \\ ${ }^{2}$ Department of Electronics and Telecommunication, Zeal Education Society, Pune 411041, India \\ e-mail: hemant.devkrupa@gmail.com
}

MS received 1 May 2017; revised 8 July 2017; accepted 19 July 2017; published online 14 March 2018

\begin{abstract}
Medical treatment and diagnosis require information that is taken from several modalities of images like Magnetic Resonance Imaging (MRI), Computerized Tomography and so on. The information obtained for certain ailments is often incomplete, invisible and lacking in consistent scanner performance. Hence, to overcome these issues in the image modalities, image fusion schemes are developed in the literature. This paper proposes a hybrid algorithm using fuzzy concept and a novel P-Whale algorithm, called Fuzzy Whale Fusion (FWFusion), for the fusion of MRI multimodal images. Two multimodal images from MRI (T1, T1C, T2 and FLAIR) are considered as the source images, which are fed as inputs to a wavelet transform. The transform utilized converts the images into four different bands, which are fused using two newly derived fusion factors, fuzzy fusion and whale fusion, in a weighted function. The proposed P-Whale approach combines Whale Optimization Algorithm (WOA) and Particle Swarm Optimization (PSO) for the effective selection of whale fusion factors. The performance of FWFusion model is compared to those of the existing strategies using Mutual Information $(M I)$, Peak Signal-to-Noise Ratio (PSNR) and Root Mean Squared Error (RMSE), as the evaluation metrics. From the mean performance evaluation, it is observed that the proposed approach can achieve $M I$ of 1.714, RMSE of 1.9 and PSNR of 27.9472.
\end{abstract}

Keywords. Image fusion; optimization; wavelet transform; fuzzy fusion factor; whale fusion factor.

\section{Introduction}

Due to the recent advancements in technology and instrumentation, medical image fusion has become an important technique that plays a major role in research, medical diagnosis and treatment $[1,2]$. In medical imaging, images of internal body features can be obtained by using an invasive or non-invasive method. To get precise clinical information, medical images that are suggested by the physicians are considered for treatment and diagnosis [3-5]. In medical image processing and analysis, for improved disease diagnosis and surgical process assisted with a computer, radiologists use medical images of high resolution with information, like tissue, region and visualization. As the design of each imaging process is made in a way to observe only certain features of the human anatomy, the requirements of medical images cannot be solved using single-modality images. It requires complementary information from multiple modalities, to diagnose the disease efficiently. Therefore, presently, a fusion of multiplemodality medical images is being used as a promising and challenging area of research [6].

*For correspondence
Medical image fusion [7, 8] is an emerging field that produces a single image having relevant information of original images by incorporating information taken from two or more images of varying modalities, without generating any noise or artefact [9]. The fused image can describe the view much better than any individual image. The fundamental objective of image fusion is to form a complementary image that contains more visual image content so that it makes the analysis more informative and thus, can be used for various medical or other applications of image processing. The approaches of image fusion are generally classified into pixel level, region level and feature level [10]. Most of the existing systems had used pixel level fusion techniques [11]. In region level schemes, pixel level fusion is employed after decomposing the image into regions. The feature level fusion methods are used broadly in data fusion process in addition to image fusion process [9]. The image fusion process can be performed in two domains, such as spatial and transform domains. In fusion techniques performed in the spatial domain, the desired result of the fusion is obtained depending on the attributes and the pixel values. The key advantage of using this domain is its ability to preserve the originality of the pixels that represent the shape more accurately. The introduction 
of spatial and spectral distortion in the resulting fused image is its only limitation. Alternatively, fusion in transform domain includes decomposing the source image into sub-image bands and then, processing selectively using a suitable fusion approach. Different transforms, such as curvelets, wavelets, Laplacian pyramid, contourlets and so on, are used for the decomposition [12].

Magnetic Resonance Imaging (MRI) is used commonly for the recognition of tumour region and bone structure, in medical image processing and analysis [6]. MRI images have different modalities that contain complementary information. This complementary information is transformed into a single image for quick and accurate diagnosis. The fusion techniques for MRI images mostly deal with wavelet transformation. In wavelet transform technique [3], the image is decomposed into a sequence of sub-band images having varying resolutions, directional characteristics and frequencies. Wavelet transforms depend on multi-resolution techniques that deal with image analysis at various resolution levels and thus, the missed feature at a level can be attained easily at another level. Discrete Wavelet Transform (DWT) is employed in Yang et al [13], which performs down-sampling during decomposition, whereas upsampling is done at reconstruction.

Although image fusion at pixel level is easy and effortless for the implementation, it suffers from major pitfalls results such as information loss and blurring of edges that demeans the quality of fused image. Thus, this paper aims to design a fusion method for MRI multimodal images using a hybrid technique, Fuzzy Whale Fusion (FWFusion), obtained by the combined effect of fuzzy weighted fusion formula and P-Whale optimizer. Two images from different modalities of MRI (T1, T1C, T2 and FLAIR) are considered for the fusion. Fusion is performed by applying the two input images to the wavelet transform that provides four different bands for each image. A weighted function that combines fuzzy and whale fusion factors is used to obtain the wavelet coefficients. The fuzzy fusion factor is computed based on the distance estimated using a membership function. For the optimal selection of whale fusion factor, a novel algorithm, named P-Whale, is utilized, where the integration of Particle Swarm Optimization (PSO) modifies Whale Optimization Algorithm (WOA). The selection of whale fusion factor depends on a newly formulated fitness function of the P-Whale algorithm. Once the coefficients are evaluated, the inverse transform can create the resulting fused image.

The contributions of the proposed approach for multimodal image fusion are stated as follows:

- The introduction of a hybrid algorithm, named FWFusion, for MRI multimodal image fusion using two newly developed factors, fuzzy fusion and whale fusion, in a weight coefficient, which will be computed effectively based on fuzzy theory and optimizer.
- Designing a novel optimization approach, P-Whale that modifies WOA with the inclusion of PSO, for the feasible selection of coefficients in fusion. The selection of best solution using P-Whale requires an objective function, which is newly developed based on Mutual Information (MI), Peak Signal-to-Noise Ratio (PSNR) and Root Mean Squared Error (RMSE).

The remainder of the paper is organized as follows. Section 2 provides a review of methodologies used for the fusion of medical images in the literature along with their limitations. Section 3 explains the proposed FWFusion approach of image fusion using the hybrid algorithm with a suitable block diagram. Section 4 illustrates the results of the proposed technique with the performance evaluation. Section 5 presents the conclusion of the paper.

\section{Motivation}

\subsection{Literature review}

This section deliberates the techniques used for medical image fusion in the existing systems with each of its limitations, which are accepted as the challenges that the proposed approach attempts to overcome. Also, the optimization algorithms related to PSO are also discussed as PSO is a part of this proposed technique.

Fusion techniques: Bhavana and Krishnappa [14], developed a fusion method by pre-processing MRI and Positron Emission Tomography (PET) images and improved the quality of the images that were degraded and non-readable using spatial filtering methods. Image fusion was then carried out in the enhanced image using DWT for the brain regions having different levels of activity. Even though the technique can provide better results, the approach failed to consider multi-modality medical images other than PET and MRI images.

Another technique for fusion was presented by Kavitha and Thyagharajan [6], based on DWT and Undecimated Discrete Wavelet Transform (UDWT), which utilized Genetic Algorithm (GA) for the estimation of weights. The lack of shift variance in DWT, during image fusion, was solved using UDWT and the analysis was made with multimodality images of the brain. The quality of the fused image was enhanced with reduced error but has high computational cost.

Koley et al [15] had designed a fusion technique using multispectral MRI image to enhance the visualization of pathological and anatomical information of meningioma (MG) brain tumours by combining contourlet transform and fuzzy statistics. This technique used contourlet transform for the decomposition, whereas fuzzy entropy and regionbased fuzzy energy were utilized for fusing approximation coefficients and detailed coefficients, respectively. However, the performance of the proposed approach did not 
show a significant improvement, when compared with that of the existing Principal Component Analysis (PCA) method.

Bhateja et al [12] presented a multimodal fusion approach in two stages, combining cascaded Stationary Wavelet Transform (SWT) and Non-Sub-sampled Contourlet Transform (NSCT) domains. The approach was implemented for images obtained from two medical imaging sensor modalities, such as MRI and Computerized Tomography (CT)-Scan. With the cascaded combination of SWT and NSCT, shift variance, phase information and directionality in the resultant image were increased. Even though the redundancy is reduced with increased contrast, the overall cost of computation required for the approach is high.

The fusion scheme developed by Srivastava et al [16] was based on curvelet transform, which was a multi-scale and multidirectional transform. The transform has a property called anisotropy, and can capture the edge points, which contains useful information, in the images efficiently for the better visualization of the image structure. The authors had used local energy-based fusion rule rather than the rules based on a single pixel for an effective fusion. Therefore, the quality of the fused image can be improved with this technique, but inaccurate fusion may exist.

Vijayarajan [17] designed a fusion method using principal component averaging based on DWT for fusing CTMRI and MRI images. Traditional PCA fusion techniques evaluated principal components depending on the eigenvalues of the images. The source images were decomposed into multi-scale inputs using DWT, and the components were computed for multi-scale coefficients. The weights for the fusion rule were found by averaging of principal components of relevant decomposed elements. With the combined advantages of wavelet transform and PCA, it could perform better than the existing methodologies. However, the procedure of DWT is complicated to understand.

Lu et al [18] had presented a technique named Maximum Local Energy (MLE), to compute the low-frequency coefficients of the images, and thereby performed image fusion. Initially, the coefficients of two images were obtained using beyond wavelet transform. Then, low-frequency and highfrequency coefficients were attained using MLE and summodified Laplacian techniques, respectively. Through beyond wavelet transform, the fused image was obtained. The performance of MLE-contourlet transform is better in CT/MRI images, but the redundancy is higher.

$\mathrm{Xu}$ et al [19] developed a fusion technique for multimodal medical images adopting Discrete Fractional Wavelet (DFRWT) with the orderp. Changing the order of $p$ within the specified domain $[0,1]$, the medical images were decomposed using DFRWT. To increase the correlation between the sub-band coefficients, the approach utilized the non-sparsity character of the mode coefficients. Using weighted regional variance rule, the coefficients of all the sub-bands were fused and inverse DFRWT was utilized to produce a fused image. Even though the approach is efficient, it is difficult to separate the subjective vision of the fusion images due to the uncertainties and randomness of fused image.

\subsection{PSO-based optimization algorithms}

De et al [20] have proposed an effective meta-heuristic, named Particle Swarm Optimization-Composite Particle (PSO-CP). The proposed algorithm assists to effortlessly flee from local optima and strongly attain near-optimal point consistently, which could be witnessed from the results. Finally, the experimental results revealed that the proposed PSO-CP was superior to the basic PSO and GA.

De et al [21] had presented an effective optimization algorithm called PSO-CP to address a maritime inventory routing issue. Moreover, it incorporates non-linear relationship between fuel consumption and vessel speed for detaining the sustainability features in the formulation. The computational outcomes attained from the PSO-CP algorithm for different issues examples dominated the solution of other methods. Maiyar and Thakkar [22] developed a decision support framework with application of PSO-CP and PSO to solve and validate the mixed-integer non-linear problem (MINLP).

\subsection{Research gaps and challenges}

Numerous research contributions have been made in the literature to deal with the issue in the fusion of MRI multimodal images. In [14], a pre-processing MRI and PET has been adopted to enhance the quality of the images. However, this method failed to consider multi-modality medical images. On the other hand, the GA [6] is known for its efficiency and supports multi-objective. Nevertheless, there is no guarantee of finding global maxima and it takes time for convergence. In [15], multispectral MRI image has been developed to enhance the visualization of pathological and anatomical information. Nonetheless, it requires consistent scanner performance and a high degree of quality control. In addition, the multimodal fusion approach [12] exploits the correlation between multiple features from different modalities. Nevertheless, the synchronization between features is more complex due to their different modalities and non-linearity. In [16], the multimodal medical image fusion increases the visual quality of the images and decreases the image artefacts and noise. However, it suffers from small spatial resolution. The principal component averaging based on DWT used in [17] reduces complexity in images, but the covariance matrix is difficult to be evaluated accurately.

In addition, the MLE exploited in [18] has the advantage of expressing point features, yet noise impact in pyramid reconstruction although it suffers from noise impact in 
pyramid reconstruction. The DFRWT used in [19] produces a fused image, but it is difficult to separate the subjective vision. Moreover, the PSO and GA used in [20-22] provide easy handling of an unknown characteristic of the system and good convergence rate. Conversely, it suffers from limited real-time performance and high dependence on algorithm parameters. Some of the challenges noticed in image fusion, based on the literature study, are as follows:

- The major challenge consideration of multimodality medical images in medical image fusion is more promising yet challenging since the selection of fusion rule is very crucial.

- Even though image fusion at pixel level is simple and easy for the implementation, it results in information loss and blurring of edges $[23,24]$ that degrades the quality of fused image. Hence, it requires attention in selecting suitable fusion approach, as careless selection may provide suboptimal results.

- Another challenge in image fusion [25] is deciding how to integrate the information from images of different modalities to obtain a single composite image with all the relevant information of source images without any artefact.

- GA was employed in [14] for MRI image fusion based on wavelet transform, where the weights for the fusion were selected using the algorithm. Since it is a traditional algorithm and has issues in convergence and local optima, performing fusion depending on GA is not feasible.

\section{FWFusion: a proposed multimodal image fusion approach using hybrid fuzzy and P-Whale-based algorithm}

This section explains the proposed fusion technique, FWFusion, for MRI multimodal images by combining fuzzy theory and P-Whale optimization approach. FWFusion algorithm considers two MRI images that are applied to a two-level wavelet transform, where the images are converted into four bands, namely Low-Low (LL), LowHigh (LH), High-Low (HL) and High-High (HH). The fused image is obtained by fusing the corresponding wavelet coefficients of the images with a weighted function that utilizes fuzzy fusion and whale fusion factors. The fuzzy approach determines the fuzzy fusion factor effectively by estimating the distance between the wavelet coefficients of both the images. Meanwhile, the proposed P-Whale technique computes the factor by the optimal selection of coefficients. Finally, the coefficients for the fused image can be obtained by averaging the two factors. Taking inverse transform, the fused image for the MRI multimodal source images can be obtained. The block diagram of the proposed technique of multimodal image fusion is demonstrated in figure 1 .
Following sections provide a detailed description of the proposed technique of medical image fusion.

\subsection{Applying wavelet transform for the two source images}

In this part, the two-level wavelet transform [26] designed for the fusion of images is explained in detail. Wavelet transform, which is an extension of Fourier Transform (FT), is used due to its effectiveness in blocking artefacts during the process. It decomposes the images into two frequency components, low and high, by two processes, such as filtering and down-sampling. The low-frequency component in wavelet transform is an approximation, whereas the latter contains detailed information. In two-level transform, these two frequency components are further decomposed into four sub-bands, LL, LH, HL and HH. These frequency components are sensitive to frequencies, such that LH, HL and HH correspond to the frequencies in vertical, horizontal and diagonal directions, respectively.

Let $I(x, y)$ be the input image filtered using a Low Pass Filter (LPF) to produce a coefficient matrix $I^{L}(x, y)$ and through a High Pass Filter (HPF), to create $I^{H}(x, y)$, after down-sampling by a scaling factor 2 . The low- and the high-frequency components generated using wavelet transform are given as

$$
\begin{gathered}
I^{L}(x, y)=\sum_{i=-N}^{N} \sum_{j=-N}^{N}[I(x, y) * L(x-i, y-j)] \\
I^{H}(x, y)=\sum_{i=-N}^{N} \sum_{j=-N}^{N}[I(x, y) * H(x-i, y-j)]
\end{gathered}
$$

where $L(x-i, y-j)$ is the filter function of LPF, $H(x-i, y-j)$ is the HPF function, $N$ is the number of pixels in the image and $*$ is the convolution operator.

At the second level, the coefficient matrices are filtered and down-sampled to form the sub-bands $I^{L L}(x, y)$, $I^{L H}(x, y), I^{H L}(x, y)$ and $I^{H H}(x, y)$. These four wavelet coefficients can be formulated as

$$
\begin{array}{r}
I^{L L}(x, y)=\sum_{i=-N}^{N} \sum_{j=-N}^{N}\left[I^{L}(x, y) * L(x-i, y-i)\right] \\
I^{L H}(x, y)=\sum_{i=-N}^{N} \sum_{j=-N}^{N}\left[I^{L}(x, y) * H(x-i, y-i)\right]
\end{array}
$$

where $I^{L}(x, y)$ is the low-frequency component obtained at level 1. Similarly, the sub-bands obtained from the high-frequency component can be formulated. $I^{L L}(x, y)$ represents the approximation of input image $I(x, y)$, whereas, the remaining sub-bands contain detailed information. 


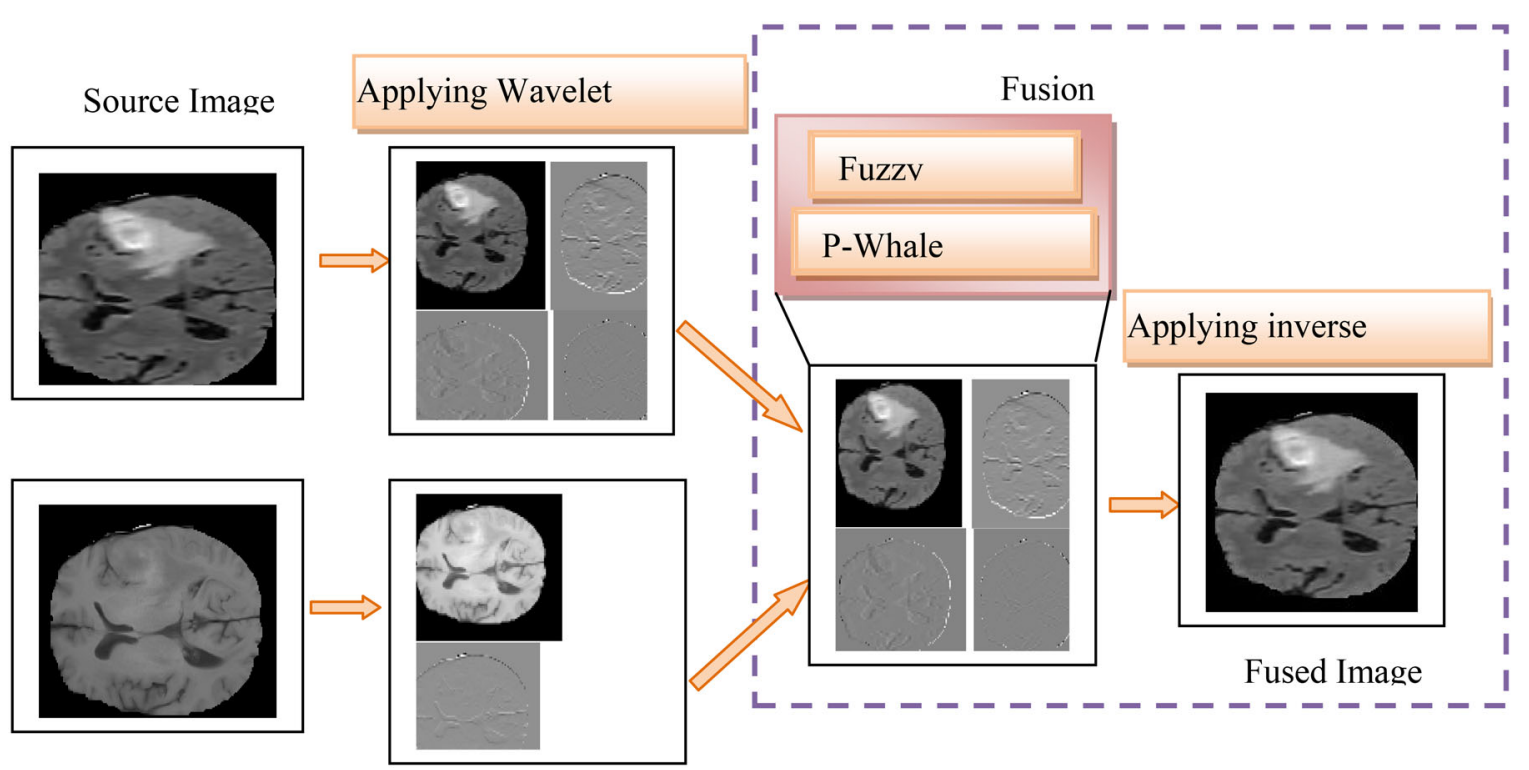

Source Image

Figure 1. Block diagram of proposed FWFusion algorithm.

In image fusion using wavelet, two source images from MRI multimodal images (T1, T1C, T2 and FLAIR), $I_{1}(x, y)$ and $I_{2}(x, y)$, are decomposed into wavelet coefficients using a fusion rule. Let $I_{1}^{L}(x, y)$ and $I_{1}^{H}(x, y)$ be, respectively, the low- and the high-frequency components of the source image $I_{1}(x, y)$, and $I_{2}^{L}(x, y)$ and $I_{2}^{H}(x, y)$ that of $I_{2}(x, y)$, which are computed using Eqs. (1) and (2), separately for both the source images. The components generated are then subjected to filtering in the vertical direction and are downsampled to form the sub-bands $I_{1}^{L L}(x, y), I_{1}^{L H}(x, y), I_{1}^{H L}(x, y)$ and $I_{1}^{H H}(x, y)$ for $I_{1}(x, y)$ and $I_{2}^{L L}(x, y), I_{2}^{L H}(x, y), I_{2}^{H L}(x, y)$ and $I_{2}^{H H}(x, y)$ for $I_{2}(x, y)$ as computed in Eqs. (3) and (4).

\subsection{Proposed fusion model for multimodal images}

Then, the coefficients for the fused image can be computed based on the corresponding coefficients of both the images using a weighted function as follows:

$$
I_{F}^{L L}(x, y)=\alpha I_{1}^{L L}(x, y)+(1-\alpha) I_{2}^{L L}(x, y)
$$

where $\alpha$ is a weight function for LL sub-band and is defined as the average of two fusion factors, as follows:

$$
\alpha=\left[\frac{\alpha_{f}+\alpha_{w}}{2}\right]
$$

where $\alpha_{f}$ is the fuzzy fusion factor and $\alpha_{w}$ is the whale fusion factor for LL sub-band.

For the LH sub-band, the function to find the fused image is formulated as

$$
I_{F}^{L H}(x, y)=\beta I_{1}^{L H}(x, y)+(1-\beta) I_{2}^{L H}(x, y)
$$

where $\beta$ is a weight function for LH sub-band, given by

$$
\beta=\left[\frac{\beta_{f}+\beta_{w}}{2}\right]
$$

where $\beta_{f}$ is the fuzzy fusion factor and $\beta_{w}$ is the whale fusion factor for LH sub-band.

The fused sub-image for HL sub-band can be computed by fusing the sub-images at that corresponding sub-band as

$$
I_{F}^{H L}(x, y)=\gamma I_{1}^{H L}(x, y)+(1-\gamma) I_{2}^{H L}(x, y)
$$

where $\gamma$ is a weight function for HL sub-band and is defined by

$$
\gamma=\left[\frac{\gamma_{f}+\gamma_{w}}{2}\right]
$$

where $\gamma_{f}$ and $\gamma_{w}$ are, respectively, the fuzzy and whale fusion factors for HL sub-band.

Similarly, for the HH sub-band, the fusion sub-image is calculated as

$$
I_{F}^{H H}(x, y)=\delta I_{1}^{H H}(x, y)+(1-\delta) I_{2}^{H H}(x, y)
$$

where $\delta$ is the weight function for the frequency sub-band $\mathrm{HH}$ :

$$
\delta=\left[\frac{\delta_{f}+\delta_{w}}{2}\right]
$$

where $\delta_{f}$ and $\delta_{w}$ are, respectively, the fuzzy fusion and whale fusion factors for HL sub-band. 


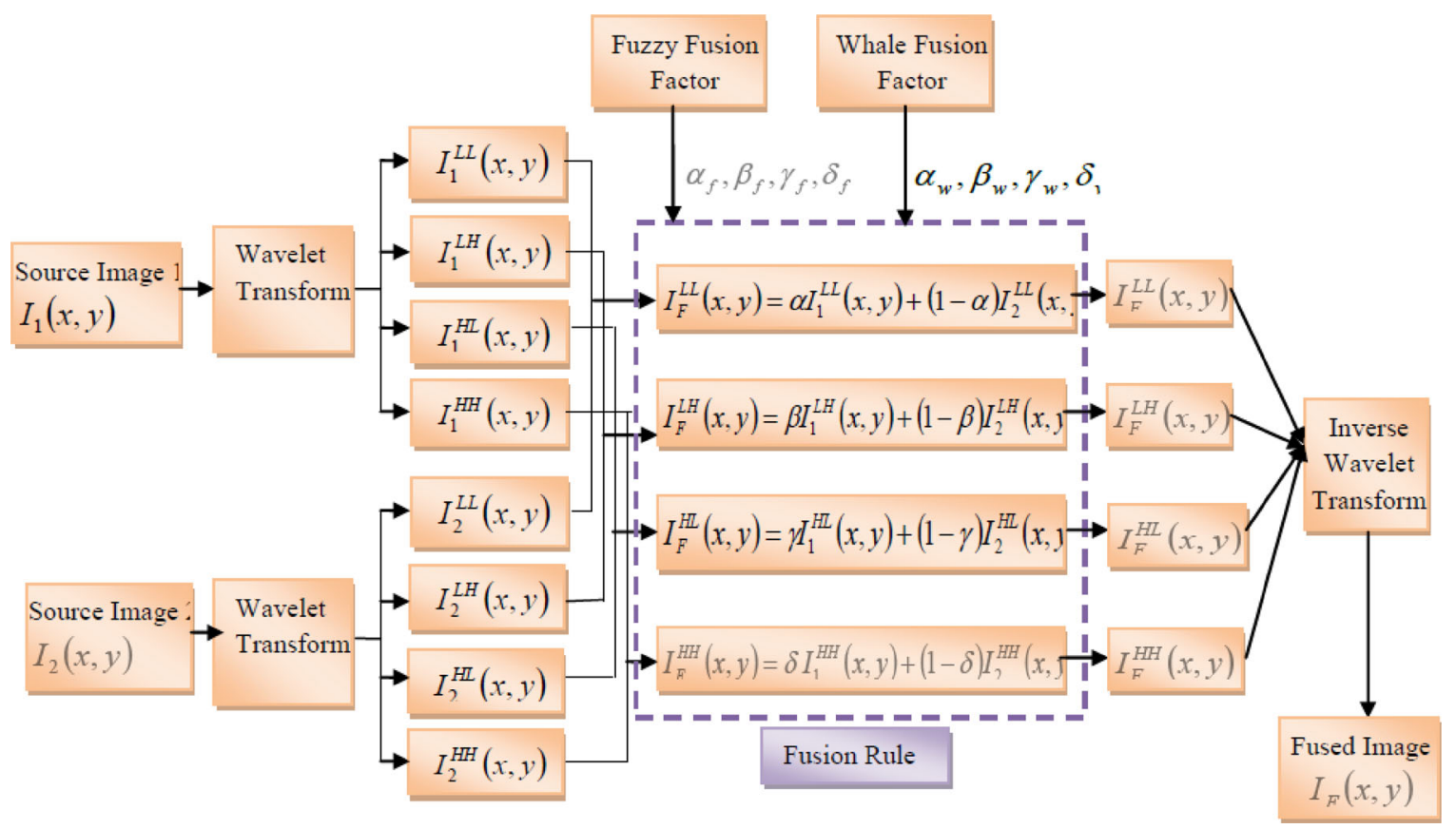

Figure 2. Model of the proposed fusion process.

These two factors are determined based on a fuzzy theory concept and a newly proposed P-Whale optimizer, which will be discussed in the following sections. Finally, the inverse wavelet transform is taken over the fused sub-images to find the fused image $I_{F}(x, y)$. The proposed fusion process is depicted clearly in figure 2 .

\subsection{Derivation of fuzzy fusion factor}

The selection process of weighted functions to develop the fused sub-images is presented in this section, using fuzzy theory. One of the main contributions of this proposed image fusion process is the evaluation of fuzzy fusion factor. The fuzzy approach improves the visualization of fused image by enhancing the resolution of the images. After the decomposition of the source images by wavelet transform, the wavelet coefficients are fused using the novel weighted function, which performs averaging of fuzzy and whale fusion factors. It requires a distance measure that computes the difference between the subbands of both the images, which will be evaluated using certain parameters using a triangular membership function. The distance $D$ between the two image sub-bands at low frequency can be computed as

$$
D_{L L}=\left\|I_{1}^{L L}(x, y)-I_{2}^{L L}(x, y)\right\|
$$

where $\|$.$\| represents the absolute value of the difference$ between the image sub-bands $I_{1}^{L L}(x, y)$ and $I_{2}^{L L}(x, y)$. For the sub-band LH of the fused image, the distance measure is given based on the difference between the matrices $I_{1}^{L H}(x, y)$ and $I_{2}^{L H}(x, y)$, as follows:

$$
D_{L H}=\left\|I_{1}^{L H}(x, y)-I_{2}^{L H}(x, y)\right\| .
$$

Similarly, for the high-frequency sub-bands, $\mathrm{HL}$ and $\mathrm{HH}$, the distance is estimated by measuring the particularly required image sub-bands:

$$
\begin{aligned}
D_{H L} & =\left\|I_{1}^{H L}(x, y)-I_{2}^{H L}(x, y)\right\|, \\
D_{H H} & =\left\|I_{1}^{H H}(x, y)-I_{2}^{H H}(x, y)\right\| .
\end{aligned}
$$

Thus, the distance measure for each image sub-band can be computed, based on which the membership function is designed, as shown in figure 3.

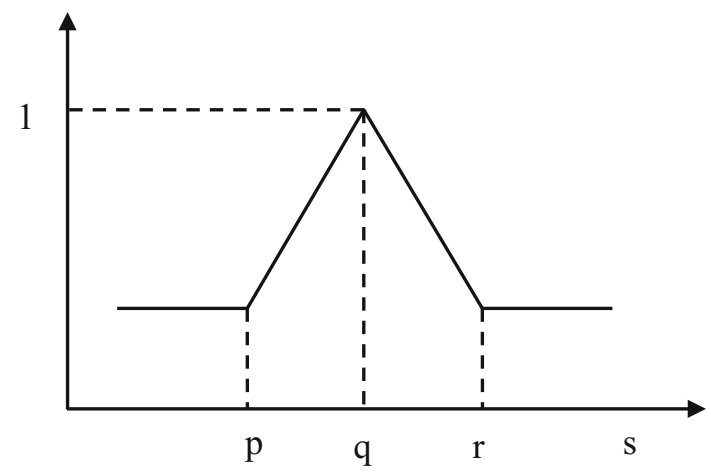

Figure 3. Triangular membership function. 
Considering four parameters, $p, q, r$ and $s$, which are fixed to desired values within the limit of $D$, i.e., $1 \leq p, q, r \leq D$ and $s=D$. Hence the fuzzy fusion factor can be determined for each sub-band of the images, based on the parameters of triangular membership function, as follows:

$$
\alpha_{f}=\left\{\begin{array}{ll}
0 ; & \text { if } s<p \\
\frac{s-p}{q-p} ; & \text { if } p<s<q \\
\frac{s-p}{r-q} ; & \text { if } q<s<r \\
0 ; & \text { if } r>s
\end{array} .\right.
$$

The fuzzy fusion factor $\alpha_{f}$ takes a value 0 if distance value is either less than $p$ or $r$ and for the other cases, the value is determined based on the formula provided. Similarly, for the other frequency sub-bands, the fuzzy fusion factor is computed based on the corresponding distance measures. Therefore, $\alpha_{f}=\beta_{f}=\gamma_{f}=\delta_{f}$, such that the value of $s$ is $D_{L L}$ for LL sub-band, $D_{L H}$ for LH sub-band, $D_{H L}$ for HL sub-band and $D_{H H}$ for $\mathrm{HH}$ sub-band image. Hence, using the proposed evaluation factor, i.e., fuzzy fusion factor, an effective fusion of MRI multimodal images can be obtained.

\subsection{Generation of whale fusion factor using the proposed $P$-Whale algorithm}

In this section, the second contribution of selecting whale fusion factor is presented using the proposed P-Whale algorithm. P-Whale is an optimization algorithm that modifies WOA by the integration of PSO in the update rule to improve the search process, and thereby enhance the performance of the algorithm. WOA [27] is a metaheuristic algorithm that solves optimization problems $[28,29]$ inspired by the physical behaviour of humpback whales, encircling prey, bubble-net attack mechanism and search for prey. In the position update of WOA, the update rule of PSO is adopted, and thus determines a suitable whale fusion factor for each sub-band. For the optimal selection of the factor, a multi-objective fitness function is formulated utilizing three objectives based on statistical evidence, visual evidence and error estimate. A detailed description of the proposed algorithm to determine the whale fusion factor for the medical image fusion is illustrated using the solution encoding, fitness function formulation and the algorithm, in following divisions.

3.4a Solution encoding: The solution representation of the proposed $\mathrm{P}-$ Whale algorithm is illustrated in this section, to describe the procedure of the algorithm in finding the fusion factor that drives the fusion process. The aim of the proposed approach is to compute an optimal whale fusion factor to fuse two images in each sub-band by fusing the wavelet coefficients. Hence, the solution set of P-Whale is denoted as $S_{i}$ that consists of Nnumber of solutions generated in random. Each solution can be represented as an array of dimension $1 \times R$, where $R$ is the number of factors to be determined, which are $\alpha_{f}, \beta_{f}, \gamma_{f}$ and $\delta_{f}$, i.e. $R=4$. The algorithm selects the best solution among $N$ solutions based on the introduced objective function and provides the whale fusion factor, which is combined with the other fusion factor and averaged for the fusion of wavelet coefficients.

3.4b Formulation of multi-objective fitness function: The function that decides the strength or the quality of the solution is the fitness function. The fitness function of the proposed $\mathrm{P}-\mathrm{Whale}$ approach considers three objectives to evaluate the fitness of the solution. The algorithm utilizes three objectives that act as functions providing statistical evidence, visual evidence and error minimization. For the statistical evidence, which provides the degree of certainty, $M I$ is included in the fitness evaluation. Visual evidence is provided regarding the PSNR and error estimate is based on $R M S E$. Hence, the objective function of the P-Whale algorithm with the utilization of MI, PSNR and RMSE [6] is formulated as follows:

$$
F_{N}=\frac{1}{3}\left[f_{S}+f_{V}+f_{E}\right]
$$

where $f_{S}$ is the fitness providing statistical evidence, $f_{V}$ is the fitness regarding visual evidence and $f_{E}$ is the fitness for the error estimation.

MI: MI, which provides the statistical evidence, gives the measure of information produced from the fusion of source images, as follows:

$$
f_{S}=\frac{1}{C_{M}}\left\{M I\left[I_{1}(x, y)-I_{F}(x, y)\right]-M I\left[I_{2}(x, y)-I_{F}(x, y)\right]\right\}
$$

where $C_{M}$ is the normalizing factor, $I_{F}(x, y)$ is the fused image, $I_{1}(x, y)$ and $I_{2}(x, y)$ are the source images and $M I[$. represents the $M I$ measured between two images, given as

$$
\begin{gathered}
M I\left[I_{1}(x, y)-I_{F}(x, y)\right]=H_{1 F} \log \frac{H_{1 F}}{H_{1} H_{F}} \\
M I\left[I_{2}(x, y)-I_{F}(x, y)\right]=H_{2 F} \log \frac{H_{2 F}}{H_{2} H_{F}}
\end{gathered}
$$

where $H_{1}$ is the histogram of the source image $I_{1}(x, y), H_{2}$ is the histogram of $I_{2}(x, y)$ and $H_{F}$ is the histogram of $I_{F}(x, y) ; H_{1 F}$ and $H_{2 F}$ are the joint histograms. Maximum $M I$ indicates higher performance in fusion. However, the function $f_{S}$ is to be minimal, as it causes the fitness function to be minimal. Hence, with the normalizing factor, the function determining statistical evidence has a value in the interval $[0,1]$.

PSNR: As defined in Eq. (22), PSNR refers to the ratio of the maximum pixel value in an image to the noise or the 
error obtained during fusion, which affects the reliability. This measure provides the visual evidence to evaluate the performance of fusion:

$f_{V}=\frac{1}{C_{V}}\left\{\operatorname{PSNR}\left[I_{1}(x, y)-I_{F}(x, y)\right]-\operatorname{PSNR}\left[I_{2}(x, y)-I_{F}(x, y)\right]\right\}$

where $C_{V}$ is the factor that normalizes the function to be in a range between 0 and 1. PSNR[.] is the PSNR computed between the source image and the fused image and is measured as

$$
P S N R=10 \log _{10} \frac{\max (\mathrm{P})^{2}}{M S E}
$$

where $P$ is the maximum pixel value in the image and $M S E$ is an error measure that computes the average of the squares of errors.

RMSE: Another objective that influences the fitness function is RMSE, which indicates good performance when it is low. This function is given as

$f_{E}=\frac{1}{C_{E}}\left\{\operatorname{RMSE}\left[I_{1}(x, y)-I_{F}(x, y)\right]-\operatorname{RMSE}\left[I_{2}(x, y)-I_{F}(x, y)\right]\right\}$

where $C_{E}$ is the normalizing factor used to normalize the function in the range $[0,1]$. The RMSE value between two images can be computed based on their differences as follows:

$$
\begin{aligned}
R M S E= & \frac{1}{2}\left(\sqrt{\frac{1}{X Y} \sum_{x=1}^{X} \sum_{y=1}^{Y}\left[I_{F}(x, y)-I_{1}(x, y)\right]^{2}}\right. \\
& \left.+\sqrt{\frac{1}{X Y} \sum_{x=1}^{X} \sum_{y=1}^{Y}\left[I_{F}(x, y)-I_{2}(x, y)\right]^{2}}\right)
\end{aligned}
$$

where $X$ and $Y$ are, respectively, the number of rows and columns in the source images $I_{1}(x, y)$ and $I_{2}(x, y)$.

With the three afore-formulated objectives, the fitness function is developed with an objective of minimization such that the solution having minimum values of $M I, P S N R$ and RMSE forms the best solution. Thus, it helps the proposed algorithm to determine the feasible factor for the weighted function to fuse the wavelet coefficients.

3.4c P-Whale algorithm: This section presents the proposed P-Whale algorithm employed for the selection of whale fusion factor. The algorithm is designed by hybridizing WOA with another optimization approach, PSO, to boost up the performance of WOA solving optimization problems. PSO simulates the social behaviour of bird flocks or fish schools. The selection of fusion factors for each subband is based on a fitness function that considers three objectives, like $M I, P S N R$ and RMSE. The procedure involved in the proposed P-Whale algorithm is described using the steps as follows.

\section{Population initialization}

The algorithm starts with the random initialization of a whale population of $\operatorname{dimension} N$, represented as

$$
U=\left\{U_{1}, U_{2}, \ldots, U_{N}\right\}, \quad 1 \leq i \leq N
$$

where $U_{i}$ represents the $i^{\text {th }}$ solution of size $R$ in the population $U$.

\section{Fitness evaluation}

After the initialization of population, the fitness of the solutions in the population is evaluated using the fitness function developed in section 3.3b. As the position of the search agents is unknown initially, the solution having the best fitness in the current population will be considered as the best agent. After defining the feasible solution, the positions of other solutions get updated based on the behaviour represented in the next step.

\section{Position update}

The position update of the P-Whale algorithm follows the encircling prey model of WOA. This requires the computation of distance between the positions of the current best agent and the vector based on a vector, given as follows:

$$
\vec{A}=\left|\vec{K} \cdot \vec{U}^{*}(t)-\vec{U}(t)\right|
$$

where $\vec{K}$ is a coefficient vector, $\vec{U}^{*}(t)$ is the position of the current best solution at iteration $t$ and $\vec{U}(t)$ is the position vector at $t$. The coefficient vector $\vec{K}$ for a random vector is given as

$$
\vec{K}=2 \cdot \vec{c}
$$

where $\vec{c}$ is the vector that assumes a random value in the range $[0,1]$.

Now, the positions of the solutions can be updated at iteration $t+1$, based on the distance vector and another coefficient vector, as

$$
\vec{U}(t+1)=\vec{U}^{*}(t)-\vec{M} \cdot \vec{A}
$$

where $\vec{A}$ is the distance vector and $\vec{M}$ is another coefficient vector, which is given as

$$
\vec{M}=2 \vec{g} \cdot \vec{c}-\vec{g}
$$

where $\vec{g}$ is a parameter that reduces linearly from 2 to 0 over the iterations.

In the exploration phase, the algorithm follows a bubblenet attacking model that involves two mechanisms, shrinking encircling and spiral update models. The first mechanism is achieved based on Eq. (30), which fixes the value of $\vec{M}$ in the range $[-g, g]$. Assigning the range as 
$[-1,1]$, the new position of the agent can be obtained. The spiral update model formulates the position using the helixshaped movement of whales by computing the distance between the agents located at two different positions:

$$
\vec{U}(t+1)=\vec{A}^{\prime} \cdot e^{d h} \cos (2 \pi h)+\vec{U}^{*}(t)
$$

where $\vec{A}^{\prime}$ is the distance measure, given by $\vec{A}^{\prime}=\left|\vec{U}^{*}(t)-\vec{U}(t)\right|, d$ is a constant that defines the shape of the spiral and $h$ is a number chosen randomly within $[-1,1]$. Hence, the position update of WOA will be based on probability $x$, adopting Eq. (29) if $x<0.5$ and Eq. (31) if $x \geq 0.5$.

In P-Whale, Eqs. (29) and (31) are modified using PSO [30] to enhance the search space. The evolution process of PSO is based on particle update using the personal best and global best positions of the swarm so that the convergence becomes faster. The position update of the swarm at iteration $t+1$ depends on the velocity update, which is given as

$$
\begin{aligned}
V(t+1)= & W V(t)+a_{1} b_{1}\left(k_{p}(t)-U(t)\right) \\
& +a_{2} b_{2}\left(k_{g}(t)-U(t)\right)
\end{aligned}
$$

where $k_{p}(t)$ is the personal best position at iteration $t, k_{g}(t)$ is the global best position at current iteration, $W$ is the inertia weight, $a_{1}$ and $a_{2}$ are the acceleration rates, $b_{1}$ and $b_{2}$ are the constants with values in the range $[0,1]$ and $U(t)$ is the position of the swarm during $k^{\text {th }}$ iteration. Hence, the position update of PSO can be derived as follows:

$$
U(t+1)=U(t)+V(t+1)
$$

Substituting Eq. (32) in (33), the update equation of PSO is

$$
\begin{aligned}
U(t+1)= & U(t)+W V(t)+a_{1} b_{1}\left(k_{p}(t)-U(t)\right) \\
& +a_{2} b_{2}\left(k_{g}(t)-U(t)\right)
\end{aligned}
$$

From this equation, the position vector at iteration $t$ is derived, which will be substituted in the position update equation of WOA, to design the update rule for the $\mathrm{P}$ Whale optimizer.

$$
\begin{gathered}
\begin{aligned}
U(t+1)= & U(t)\left[1-a_{1} b_{1}-a_{2} b_{2}\right]+W V(t)+a_{1} b_{1} k_{p}(t) \\
& +a_{2} b_{2} k_{g}(t),
\end{aligned} \\
\begin{aligned}
U(t)\left[1-a_{1} b_{1}-a_{2} b_{2}\right]= & U(t+1)-W V(t)-a_{1} b_{1} k_{p}(t) \\
& -a_{2} b_{2} k_{g}(t),
\end{aligned} \\
U(t)=\frac{1}{\left[1-a_{1} b_{1}-a_{2} b_{2}\right]} \\
{\left[U(t+1)-W V(t)-a_{1} b_{1} k_{p}(t)-a_{2} b_{2} k_{g}(t)\right] .}
\end{gathered}
$$

As the global best position in PSO is equivalent to the position of the best solution, it will be replaced with the best position vector, i.e., $k_{g}(t)=\vec{U}^{*}(t)$.

Update rule of $P$-Whale: To develop the update rule of the proposed P-Whale algorithm, the position update equations of both WOA and PSO are combined. Substituting Eq. (37) in (29) with its distance measure substituted, the position update of P-Whale based on shrinking mechanism can be derived as follows:

$$
\begin{aligned}
\vec{U}(t+1)= & \vec{U}^{*}(t)-\vec{M} \cdot \vec{K} \cdot \vec{U}^{*}(t)+\vec{M}\left[\frac{1}{\left[1-a_{1} b_{1}-a_{2} b_{2}\right]}\right. \\
& {\left.\left[U(t+1)-W V(t)-a_{1} b_{1} k_{p}(t)-a_{2} b_{2} \vec{U}^{*}(t)\right]\right] }
\end{aligned}
$$

$$
\begin{gathered}
\vec{U}(t+1)=\vec{U}^{*}(t)[1-\vec{M} \cdot \vec{K}]+\frac{\vec{M}}{\left[1-a_{1} b_{1}-a_{2} b_{2}\right]} U(t+1) \\
-\frac{\vec{M}}{\left[1-a_{1} b_{1}-a_{2} b_{2}\right]}\left[W V(t)+a_{1} b_{1} k_{p}(t)+a_{2} b_{2} \vec{U}^{*}(t)\right]
\end{gathered}
$$

$$
\begin{aligned}
& \vec{U}(t+1)=\frac{1-a_{1} b_{1}-a_{2} b_{2}}{1-a_{1} b_{1}-a_{2} b_{2}-\vec{M}}\left[\vec{U}^{*}(t)[1-\vec{M} \cdot \vec{K}]\right. \\
& \left.-\frac{\vec{M}}{\left[1-a_{1} b_{1}-a_{2} b_{2}\right]}\left[W V(t)+a_{1} b_{1} k_{p}(t)+a_{2} b_{2} \vec{U}^{*}(t)\right]\right]
\end{aligned}
$$

where $k_{p}(t)$ is the personal best position at iteration $t, W$ is the inertia weight, $a_{1}$ and $a_{2}$ are the acceleration rates, $b_{1}$ and $b_{2}$ are constants, $\vec{M}$ and $\vec{K}$ are the coefficient vectors and $\vec{U}^{*}(t)$ is the position of the best solution at $t^{\text {th }}$ iteration.

Adopting the spiral model, the update equation of $\mathrm{P}$ Whale is given by substituting Eq. (37) in (31) with the value of $\vec{A}^{\prime}$ substituted, as follows:

$$
\begin{gathered}
\vec{U}(t+1)=\vec{U}^{*}(t) e^{d h} \cos (2 \pi h)+\vec{U}^{*}(t)-\frac{e^{d h} \cos (2 \pi h)}{\left[1-a_{1} b_{1}-a_{2} b_{2}\right]} \\
{\left[U(t+1)-W V(t)-a_{1} b_{1} k_{p}(t)-a_{2} b_{2} \vec{U}^{*}(t)\right]}
\end{gathered}
$$

$$
\begin{aligned}
& \vec{U}(t+1)+\frac{e^{d h} \cos (2 \pi h)}{\left[1-a_{1} b_{1}-a_{2} b_{2}\right]} U(t+1) \\
& =\vec{U}^{*}(t)\left[1+e^{d h} \cos (2 \pi h)\right]+\frac{e^{d h} \cos (2 \pi h)}{\left[1-a_{1} b_{1}-a_{2} b_{2}\right]} \\
& \quad\left[W V(t)+a_{1} b_{1} k_{p}(t)+a_{2} b_{2} \vec{U}^{*}(t)\right] \\
& \vec{U}(t+1)=\frac{1-a_{1} b_{1}-a_{2} b_{2}}{1-a_{1} b_{1}-a_{2} b_{2}+e^{d h} \cos (2 \pi h)} \\
& \left\{\vec{U}^{*}(t)\left[1+e^{d h} \cos (2 \pi h)\right]+\frac{e^{d h} \cos (2 \pi h)}{\left[1-a_{1} b_{1}-a_{2} b_{2}\right]}\right. \\
& \left.\left[W V(t)+a_{1} b_{1} k_{p}(t)+a_{2} b_{2} \vec{U}^{*}(t)\right]\right\}
\end{aligned}
$$


where $d$ is a constant defining the shape of the spiral model, and $h$ is a random number. Eq. (44) is the update equation of P-Whale when $x \geq 0.5$. Hence, the mathematical model of the position update of $\mathrm{P}-\mathrm{Whale}$ can be represented as
Dataset description: the database considered for the experimentation is Multimodal BRAin Tumor image Segmentation (BRATS) MRI [31], which consists of training datasets in Virtual Skeleton Database (VSD). The dataset consists of data

$\vec{U}(t+1)=\left\{\begin{array}{c}\frac{1-a_{1} b_{1}-a_{2} b_{2}}{1-a_{1} b_{1}-a_{2} b_{2}-\vec{M}}\left[\vec{U}^{*}(t)[1-\vec{M} \cdot \vec{K}]-\frac{\vec{M}}{\left[1-a_{1} b_{1}-a_{2} b_{2}\right]}\left[W V(t)+a_{1} b_{1} k_{p}(t)+a_{2} b_{2} \vec{U}^{*}(t)\right]\right] \\ \begin{array}{c}\text { if } x<0.5, \\ \frac{1-a_{1} b_{1}-a_{2} b_{2}}{1-a_{1} b_{1}-a_{2} b_{2}+e^{d h} \cos (2 \pi h)}\left\{\vec{U}^{*}(t)\left[1+e^{d h} \cos (2 \pi h)\right]+\frac{e^{d h} \cos (2 \pi h)}{\left[1-a_{1} b_{1}-a_{2} b_{2}\right]}\left[W V(t)+a_{1} b_{1} k_{p}(t)+a_{2} b_{2} \vec{U}^{*}(t)\right]\right. \\ \text { if } x \geq 0.5,\end{array}\end{array}\right.$

where $x$ is the probability within the interval $[0,1]$.

\section{Finding the best candidate solution}

Once the positions of the solutions are updated, the fitness values are computed using Eq. (18). Since the objective is fitness minimization, the solution having minimum fitness forms the best solution. At each iteration, the solution with minimal fitness replaces the previous solution, and thus, the best agent can be determined.

\section{Termination}

The steps from I to IV are repeated until a termination condition is reached. The criterion to terminate the algorithm is when the iteration $t$ reaches the maximum count. Finally, the P-Whale algorithm returns the best solution, which is the whale fusion factor.

\section{Results and discussion}

This section demonstrates the results of the proposed FWFusion algorithm used for the fusion of multimodal MRI images. Moreover, a comparative analysis is performed by comparing the performance of the proposed approach with existing algorithms and the results are also illustrated.

\subsection{Experimental set-up}

The experiment is performed in a system with Windows 8 Operating System, 2 GB RAM and Intel Core i-3 processor. The software used for the implementation of the fusion of MRI multimodal images is MATLAB.

Parametric values: The values assigned to the parameters used in the FWFusion approach are as follows: size of the population $N=10$ and membership function parameters $p=100, q=1073.5, r=2047$ and $s=2147$. from BRATS 2012 and BRATS 2013 and NIH Cancer Imaging Archive, developed as part of BRATS 2014 and BRATS 2015. Each dataset comprises T1 MRI, T1 contrast-enhanced MRI, T2 MRI and T2 FLAIR MRI volumes.

\subsection{Evaluation metrics}

The metrics used for the performance evaluation of image fusion are MI, PSNR and RMSE, which are defined in section $3.4 \mathrm{~b}$. These measures are computed by taking the average of those measured between source image 1 and fused image and between source image 2 and fused image, as follows:

$$
\begin{gathered}
M I=\frac{1}{2}\left[M I\left[I_{1}(x, y), I_{F}(x, y)\right]+M I\left[I_{2}(x, y), I_{F}(x, y)\right]\right], \\
P S N R=\frac{1}{2}\left[\operatorname{PSNR}\left[I_{1}(x, y), I_{F}(x, y)\right]+\operatorname{PSNR}\left[I_{2}(x, y), I_{F}(x, y)\right]\right],
\end{gathered}
$$

$R M S E=\frac{1}{2}\left[R M S E\left[I_{1}(x, y), I_{F}(x, y)\right]+\operatorname{RMSE}\left[I_{2}(x, y), I_{F}(x, y)\right]\right]$.

\subsection{Methods employed for comparison}

The methods that are used in the comparative analysis to compare the performance are (i) wavelet+average [17] (ii) wavelet + fuzzy + WOA (applied WOA in the proposed fusion process instead of the P-Whale algorithm) and (iii) wavelet + fuzzy + GA [6]. In [17], by performing an average of principal components, the image fusion was performed, whereas in [6], GA was used for the estimation of weights in the fusion process.

\subsection{Experimental results}

The experimental results of the proposed approach of multimodal MRI image fusion are illustrated in this 

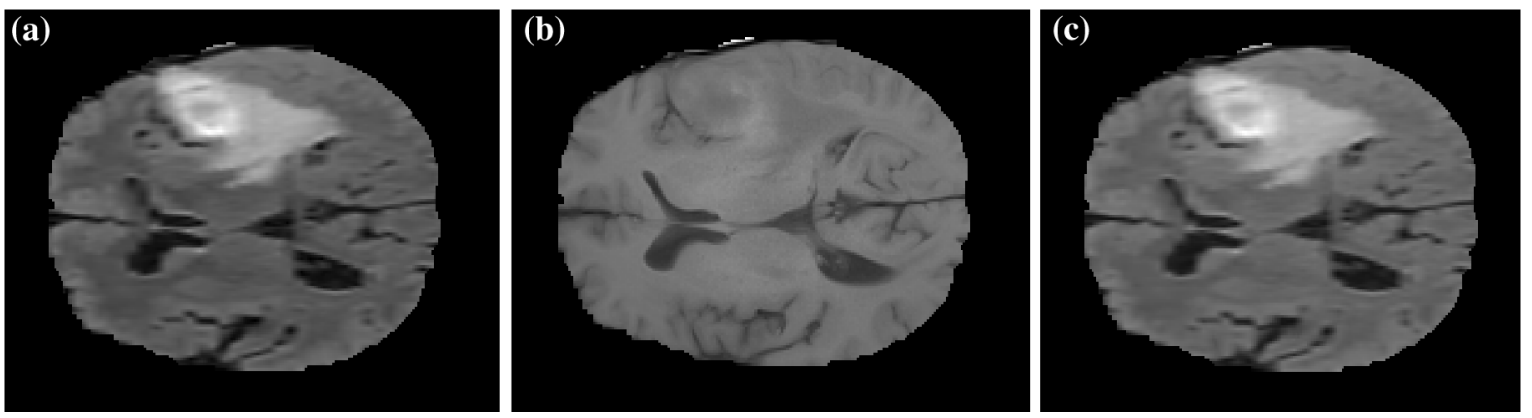

Figure 4. Fusion result using db1 wavelet transform. a T1 (source image 1). b FLAIR (source image 2). c Fused image.
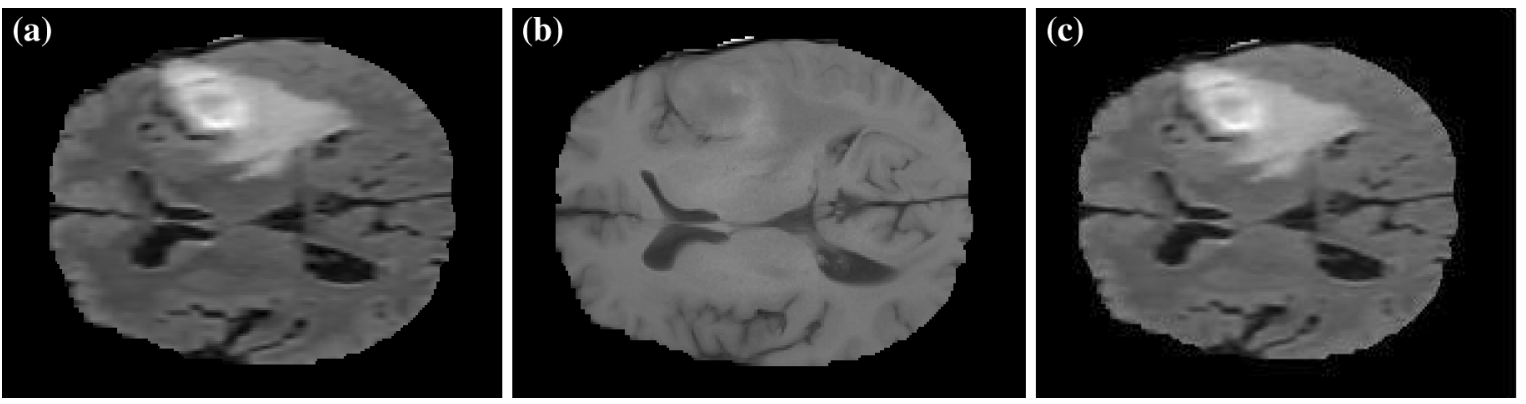

Figure 5. Fusion result using Haar wavelet transform. a T1 (source image 1). b FLAIR (source image 2). c Fused image.

section. The results of fusion using two multimodal images, $\mathrm{T} 1$ and FLAIR, from the BRATS database in two wavelet transforms, Daubechies 1 (db1) wavelet and Haar wavelet transform, are presented here. In figure 4 , the results of image fusion performed using $\mathrm{db} 1$ wavelet transform is depicted in the two MRI images of different modalities. Figure $4 \mathrm{a}$ and $\mathrm{b}$ shows, respectively, the MRI images of $\mathrm{T} 1$ and FLAIR, which are the source images to be fused. In figure $4 \mathrm{c}$, the resulting fusion image obtained using $\mathrm{db} 1$ wavelet transform with the proposed FWFusion approach, which utilizes the fusion process based on fuzzy and whale fusion factors, is given.

Figure 5 shows the fusion of two multimodal images that are fused using the Haar wavelet transform. The source MRI images T1 and FLAIR that are to be fused are pictured in figure $5 \mathrm{a}$ and $\mathrm{b}$. In figure $5 \mathrm{c}$, the image fusion result provided by the Haar wavelet with the proposed FWFusion approach is depicted. From the results shown in figures $4 \mathrm{c}$ and $5 \mathrm{c}$, using the two wavelet transforms with the proposed algorithm, it is observed that better results of fusion can be obtained with all the informative contents of the source images shown.

\subsection{Performance analysis}

The comparative analysis made to analyse the performance of the fusion process in the three comparative techniques is presented in this section. For the analysis, the metrics $M I$, $P S N R$ and RMSE are evaluated using Daubechies 2 wavelet (db2), Haar wavelet, Coiflets 1 wavelet (coif1) and Symlet Wavelet (sym2) transform.

4.5a Analysis based on MI: Figure 6 shows the performance analysis plot based on $M I$ using the four wavelets in the comparative techniques. Higher the $M I$, greater the performance. In figure $6 \mathrm{a}$, the $M I$ analysis results using $\mathrm{db} 2$ wavelet in wavelet+average, wavelet+fuzzy+WOA, wavelet+fuzzy+GA and wavelet+FWFusion with different-modality MRI images are presented. For the fusion made using FLAIR and T1 images, the MI measured using wavelet+average, wavelet+fuzzy+WOA, wavelet+fuzzy+GA and wavelet+FWFusion is 1.5701, 1.6115, 1.6169 and 1.6564, respectively. The proposed wavelet+FWFusion method has the maximum value of 1.8187 , for the fusion using $\mathrm{T} 1$ and $\mathrm{T} 2$ images, whereas the maximum $M I$ obtained in the existing wavelet+average is just 1.6785. The results of analysis based on $M I$ using Haar wavelet is depicted in figure $6 \mathrm{~b}$, where the maximum $M I$ provided by the proposed method is 1.8188 , for the fusion made using T1 and T2 images. With the same images, $M I$ calculated using wavelet+average, wavelet+fuzzy+WOA and wavelet+fuzzy+GA is $1.7684,1.4822$ and 1.4487 , respectively. Figure $6 \mathrm{c}$ depicts the comparative analysis chart based on $M I$ using coif 1 wavelet. When the images FLAIR and T1 are fused, $M I$ obtained using the existing 


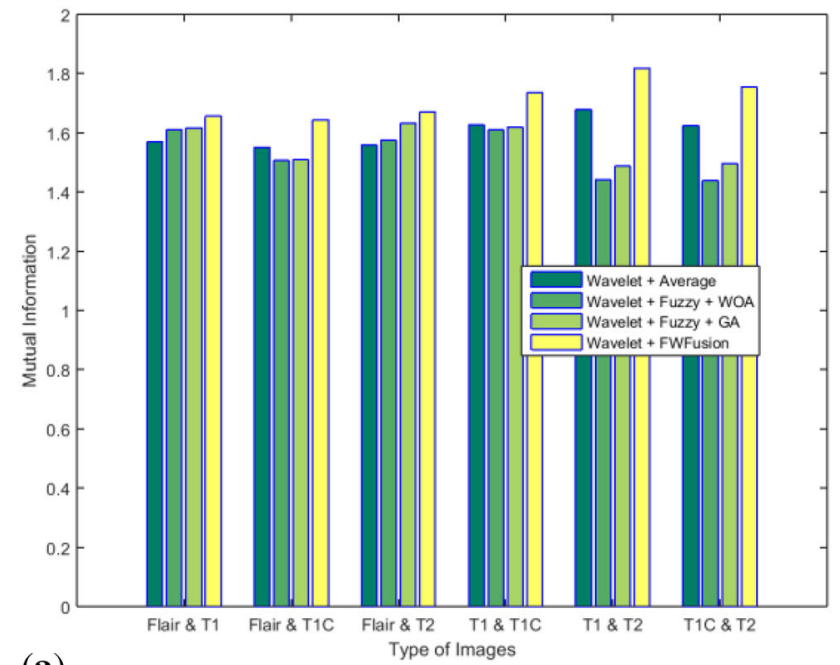

(a)

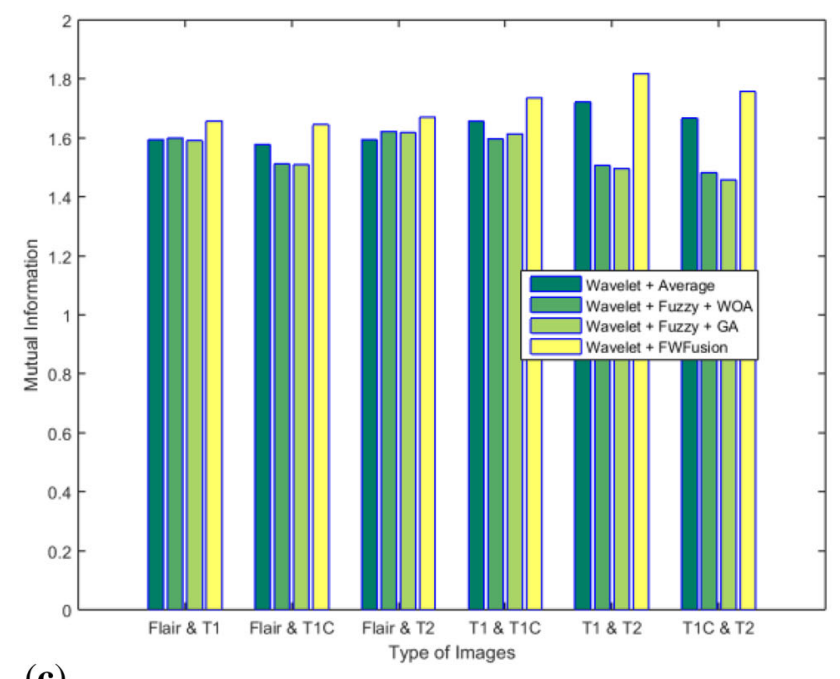

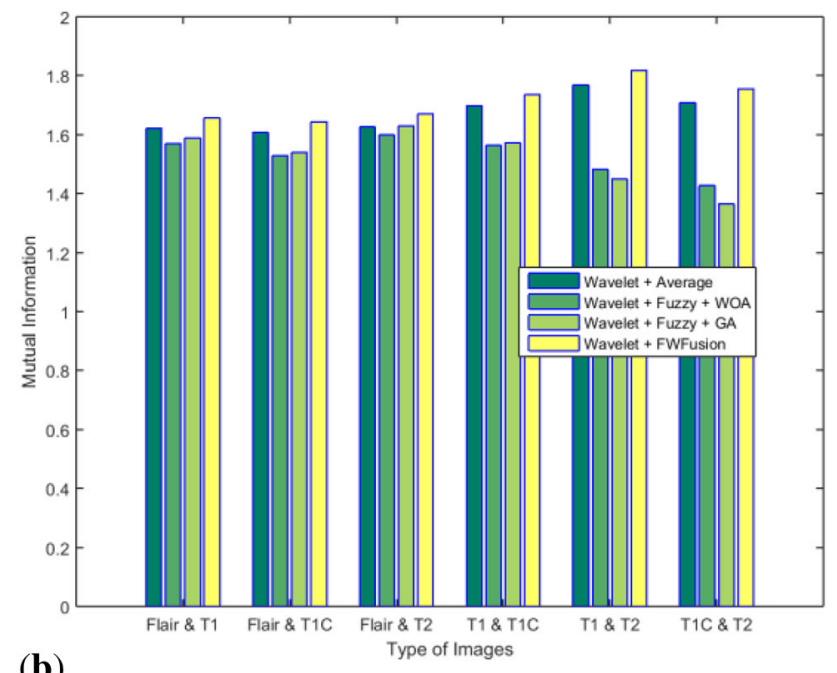

(b)

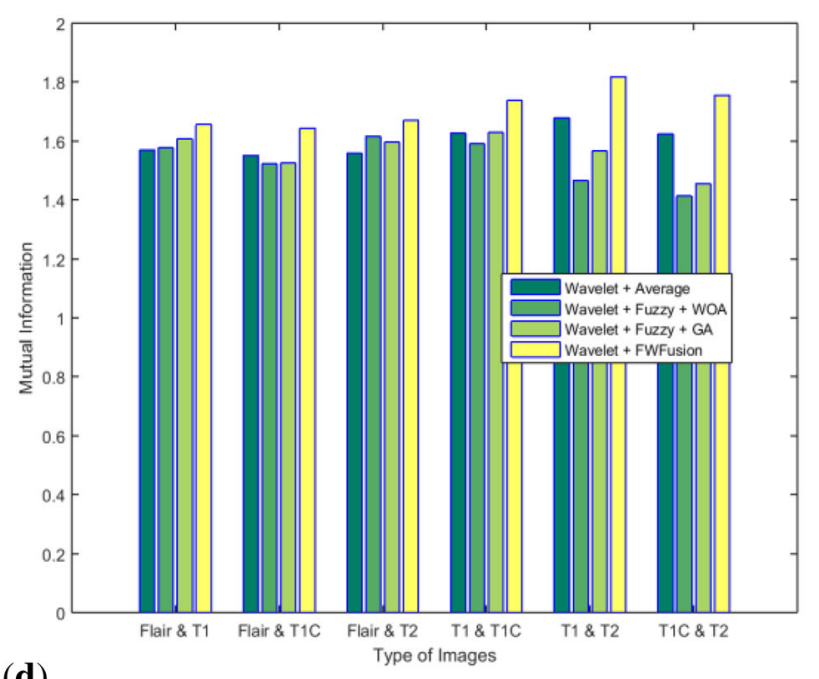

(d)

Figure 6. Performance analysis based on MI. a db2 wavelet. b Haar wavelet. c coif1 wavelet. d sym2 wavelet.

methods wavelet+average, wavelet+fuzzy+WOA and wavelet+fuzzy+GA is 1.5950, 1.6007 and 1.5907, respectively, while the proposed wavelet+FWFusion gives 1.6577 as the $M I$. The maximum $M I$ is obtained when the fusion is made using T1 and T2 images with a value of 1.8187 by wavelet+FWFusion. $M I$ analysis result obtained using sym2 wavelet is depicted in figure $6 \mathrm{~d}$ with the multimodal images FLAIR, T1, T2 and T1C. In this case too, maximum $M I$ is observed for the fusion made using $\mathrm{T} 1$ and T2 images. The maximum value attained with this fused image using wavelet+average, wavelet+fuzzy+WOA, wavelet+fuzzy+GA and wavelet+FWFusion is 1.6785 , $1.4662,1.5662$ and 1.8187 , respectively.

4.5b Analysis based on RMSE: The comparative analysis results based on $R M S E$ performed using the wavelets, $\mathrm{db} 2$, Haar, coif 1 and sym 2 in the four comparative approaches is demonstrated in figure 7 , using six combinations of fused images. An effective algorithm requires minimum RMSE for the performance improvement. In figure 7a, the RMSE analysis chart using $\mathrm{db} 2$ wavelet is shown. When the images FLAIR and T2 are fused, the RMSE values attained by the techniques wavelet+average, wavelet+fuzzy+WOA, wavelet+fuzzy+GA and wavelet+FWFusion are 2.1167, 2.9339, 2.5169 and 1.9936, respectively. Figure $7 \mathrm{~b}$ presents the RMSE analysis performed in the four approaches using the Haar transform. The minimum RMSE obtained using wavelet+average, wavelet+fuzzy+WOA and wavelet+fuzzy+GA is $1.1579,5.3770$ and 5.4939, respectively, while wavelet+FWFusion gives 1.1455 as the minimum RMSE for the combination of images T1 and T2. The analysis based on RMSE using coif1 wavelet is pictured in figure $7 \mathrm{c}$, where the minimum RMSE obtained using the proposed wavelet+FWFusion method is 1.1135 , 


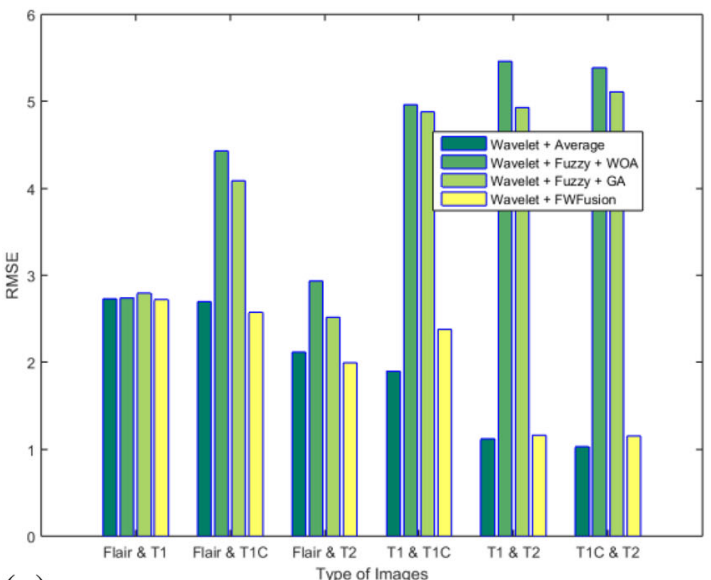

(a)

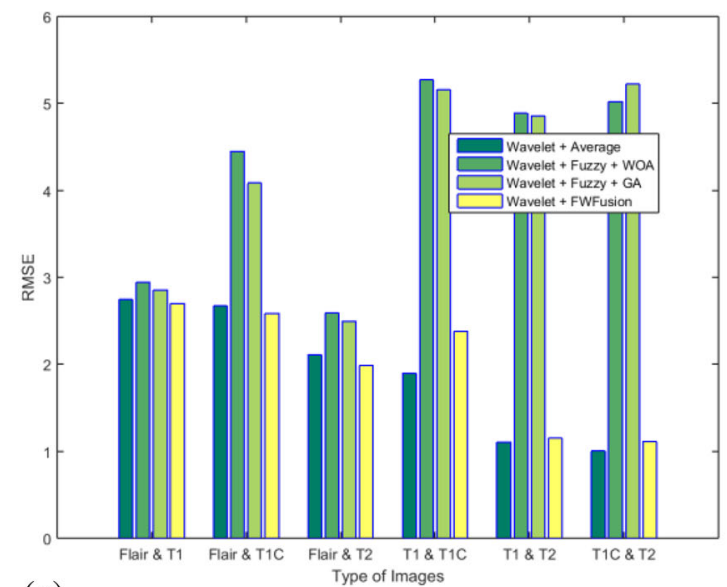

(c)

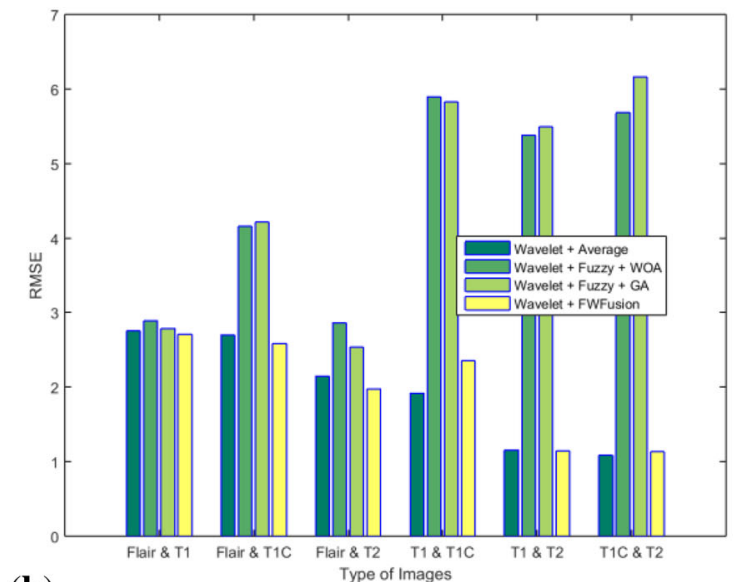

(b)

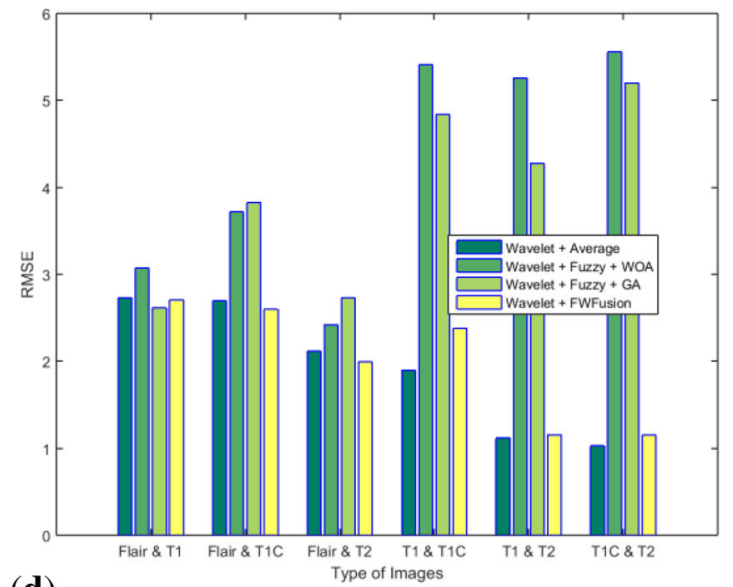

(d)

Figure 7. Performance analysis based on RMSE. a db2 wavelet. b Haar wavelet. c coif1 wavelet. d sym2 wavelet.

which is slightly more than that obtained using wavelet+average, which gives the value 1.0031 for the fusion of T1C and T2 images. However, for most of the other combinations, wavelet+FWFusion gives the least $R M S E$. In figure 7d, the RMSE analysis graph using sym2 wavelet is shown. The RMSE value obtained when the images FLAIR and T2 are fused is 2.11675, 2.4224, 2.7295 and 1.9922 using the methods wavelet+average, wavelet+fuzzy+WOA, wavelet+fuzzy+GA and wavelet+FWFusion, respectively. For the combinations $\mathrm{T} 1 \& \mathrm{~T} 1 \mathrm{C}, \mathrm{T} 1 \& \mathrm{~T} 2$ and $\mathrm{T} 1 \mathrm{C} \& \mathrm{~T} 2$, the RMSE value of the proposed approach tends to increase a little than in the existing wavelet+average, which is negligible, since the proposed approach has better performance for the other combinations. Moreover, when the performance of proposed wavelet+FWFusion is compared with wavelet+fuzzy+WOA and wavelet+fuzzy+GA, the proposed approach has attained better results.

4.5c Analysis based on PSNR: In this part, the results of $P S N R$ analysis with the techniques wavelet+average, wavelet+fuzzy+WOA, wavelet+fuzzy+GA and
wavelet+FWFusion, for different MRI image combinations, are presented in figure 8 . The performance increases with increasing $P S N R$. Figure 8a pictures out the analysis made based on PSNR using $\mathrm{db} 2$ wavelet. When FLAIR and T1 images are fused, the $P S N R$ value estimated in wavelet+average, wavelet+fuzzy+WOA, wavelet+fuzzy+GA and wavelet+FWFusion is 23.1239, 23.5644, 23.5921 and 24.9212 , respectively. This value tends to be maximum for the fused images $\mathrm{T} 1$ and $\mathrm{T} 2$ with PSNR $30.9526,19.1068,21.0467$ and 34.4518 in the techniques wavelet+average, wavelet+fuzzy+WOA, wavelet+fuzzy $+\mathrm{GA}$ and wavelet+FWFusion. In figure $8 \mathrm{~b}$, the $P S N R$ analysis plotted against different combinations of MRI images using Haar transform is illustrated. The maximum PSNR value observed in the existing techniques wavelet+average, wavelet+fuzzy+WOA and wavelet+fuzzy+GA is $30.7763,20.0139$ and 18.7884 , respectively, whereas in wavelet+FWFusion it is 34.4083 . The comparative analysis based on $P S N R$ using coif1 wavelet transform is presented in figure 8c. For the fusion made using FLAIR and T1C, the PSNR attained using wavelet+average is 22.8028, whereas in wavelet+fuzzy+WOA and 

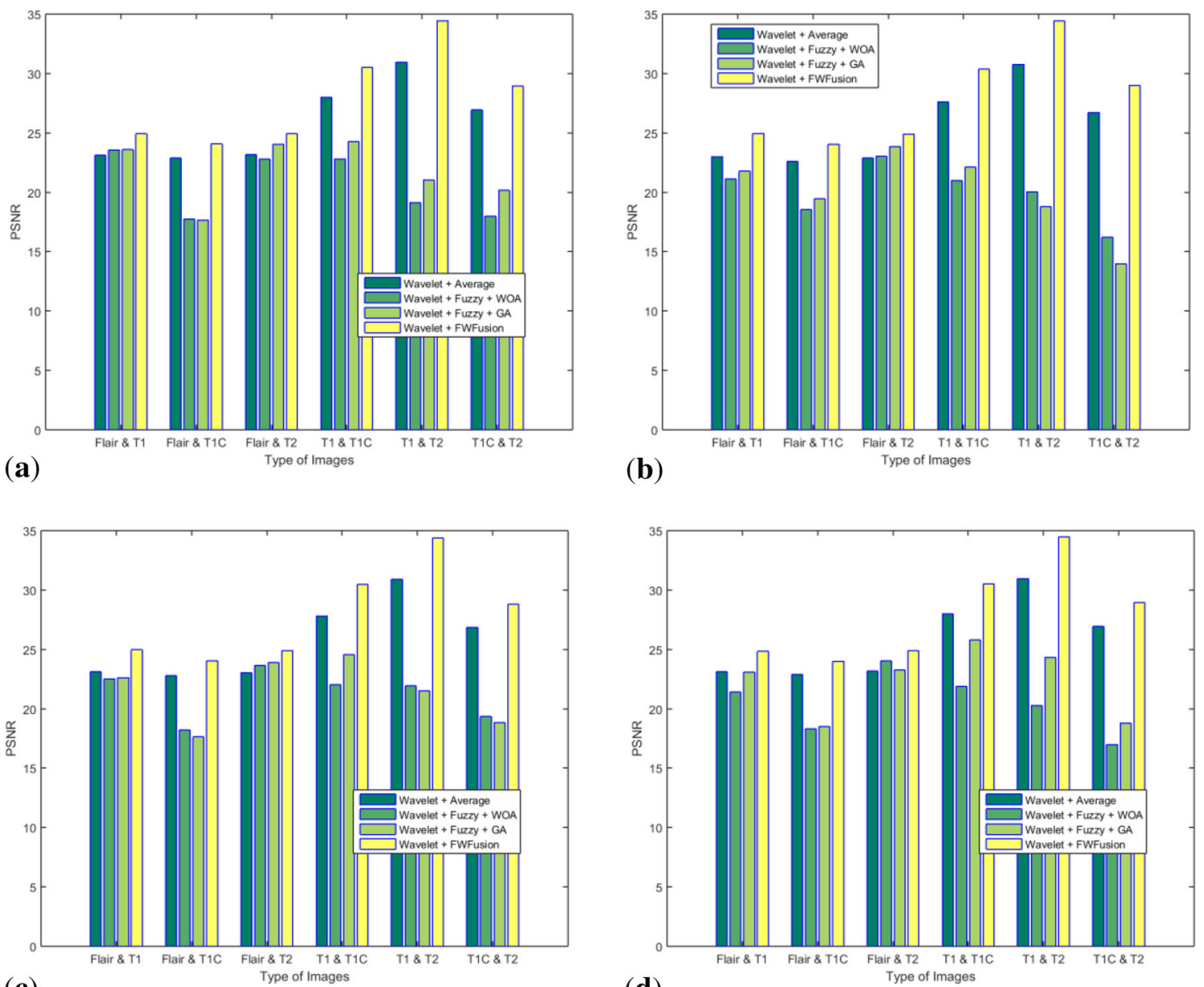

(c)

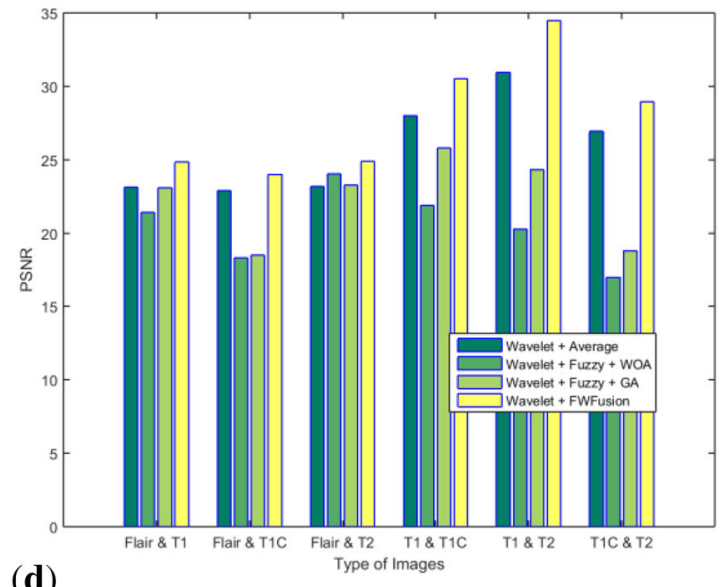

(d)

Figure 8. Performance analysis based on PSNR. a db2 wavelet. b Haar wavelet. c coif1 wavelet. d sym2 wavelet.

wavelet+fuzzy+GA it is 18.2242 and 17.6234 , respectively. In this case, the proposed wavelet+FWFusion approach can attain PSNR of 24.0338, which is $5.12 \%$ more than the PSNR from the existing wavelet+average. The maximum $P S N R$ possible in wavelet + FWFusion method using coif1 wavelet is 34.3778 when the images T1 and T2 are fused. Figure $8 \mathrm{~d}$ presents the analysis chart based on PSNR using sym2 wavelet in the four comparative techniques. When the multimodal images T1C and T2 are fused, the PSNR measured using wavelet+average, wavelet+fuzzy+WOA, wavelet+fuzzy+GA and wavelet+FWFusion is 26.9297, 16.9984, 18.7771 and 28.9463, respectively. These methods are found to have the maximum PSNR when the images T1 and T2 are fused, where a $P S N R$ of 34.4599 is possible with the proposed approach.

\subsection{Discussion}

From the overall comparative analysis result demonstrated in section 4.5, a discussion is made regarding the three evaluation metrics, MI, RMSE and PSNR. Based on these three metrics, the performance of the proposed method is compared to those of the existing methods. The $M I$ metric is exploited to measure the degree of dependence of two images. In addition, the $M S E$ and $P S N R$ are the two error metrics exploited to compare the image compression quality. In addition, an effective algorithm needs maximum $M I$ as well as PSNR and minimum RMSE. Table 1 presents the performance comparison carried out in fusion using different combinations of multimodal images by computing the mean performance for the evaluation.

As shown in table 1 , the mean values obtained using the four wavelets for the metrics MI, RMSE and PSNR in the comparative methods wavelet+average, wavelet+fuzzy+WOA, wavelet+fuzzy+GA and wavelet $+\mathrm{FWFu}-$ sion are listed. While wavelet+average had $M I$ of 1.6276, the proposed method could attain a mean value of 1.714 . It is found that the proposed wavelet+FWFusion method has better $M I$ and it performs $5.30 \%$ better than the existing wavelet+average, $11 \%$ better than the existing wavelet+fuzzy+WOA and wavelet+fuzzy+GA. The RMSE by the existing wavelet+average, wavelet+fuzzy+WOA and 
Table 1. Mean performance comparison.

\begin{tabular}{lllc}
\hline Methods & MI & RMSE & PSNR \\
\hline Wavelet+average & 1.6276 & 1.9362 & 25.7604 \\
Wavelet+fuzzy+WOA & 1.5359 & 4.3072 & 20.6021 \\
Wavelet+fuzzy+GA & 1.5488 & 4.1455 & 21.3917 \\
Wavelet+FWFusion & 1.714 & 1.9 & 27.9472 \\
\hline
\end{tabular}

wavelet+fuzzy+GA is 1.9362, 4.3072 and 4.1455, respectively, whereas wavelet+FWFusion could attain a value of 1.9. In addition, the RMSE of the proposed method is $2 \%$ better than that by the existing wavelet+average, $35 \%$ better than that by the existing wavelet+fuzzy+WOA and $54 \%$ better than that by the wavelet+fuzzy+GA. PSNR achieved by the proposed wavelet+FWFusion approach is 27.9472, whereas the existing wavelet+average had only 25.7604 as the mean PSNR. In terms of PSNR, the proposed method performance is $8.4 \%, 35 \%$ and $30 \%$ better than that of the wavelet+FWFusion, wavelet+average and wavelet+fuzzy+GA. Thus, from the discussion, it is clear that the proposed wavelet+FWFusion method has better results than the existing techniques compared.

Discussing about the better performance in terms of $M I$ metric, the proposed method shows significant improvement as compared with existing because of the dependence of pixel intensities utilized by the fuzziness concept and also the maximum utilization of flexible fusion of the information level. Also, discussing about the performance in terms of MSE and PSNR, the proposed method outperforms the existing ones because of the P-Whale optimization, which finds the optimal coefficient for fusion of two images by utilizing the visual quality as fitness.

\section{Conclusion}

In this paper, a novel FWFusion algorithm designed using fuzzy and P-Whale algorithm is presented for the fusion of MRI multimodal images. Two multimodal images taken from MRI (T1, T1C, T2 and FLAIR) are transformed into four sub-bands LL, LH, HL and $\mathrm{HH}$, using wavelet transform. The wavelet coefficients are then fused using a weighted function that introduces two fusion factors, called fuzzy fusion factor and whale fusion factor. Moreover, an optimization approach, P-Whale, is designed by modifying WOA using PSO, for the optimal selection of whale fusion factor. For the effective determination of fusion factors, a fitness function is formulated considering $M I, P S N R$ and RMSE, which provides, respectively, statistical evidence, visual evidence and error estimate. The performance of the proposed method is compared to three existing methods of fusion, wavelet+average, wavelet+fuzzy+WOA and wavelet+fuzzy+GA, using the metrics $M I, R M S E$ and $P S N R$. From the mean evaluation, the proposed fusion scheme could attain $M I$ of $1.714, R M S E$ of 1.9 and PSNR of
27.9472, while the existing wavelet+average had only 1.6276, 1.9362 and 25.7604 as the best values of MI, RMSE and PSNR. Hence, it can be concluded that the proposed FWFusion algorithm outperforms the existing fusion techniques, providing better performance.

\section{References}

[1] Wang Z, Ziou D, Armenakis C, Li D and Li Q 2005 A comparative analysis of image fusion methods. IEEE Trans. GeoSci. Remote Sens. 43(5): 1391-1402

[2] Kim Y, Lee C, Han D, Kim Y and Kim Y 2011 Improved additive-wavelet image fusion. IEEE Trans. Geosci. Remote Sens. Lett. 8(2): 263-267

[3] Goshtas A A and Nikolov S 2007 Image fusion: advances in the state of the art. Inf. Fus. 8(2): 114-118

[4] Dammavalam S R, Maddala S and Krishna Prasad M H M 2012 Comparison of fuzzy and neuro-fuzzy image fusion techniques and its applications. Int. J. Comput. Appl. 43(19): $31-37$

[5] Dammavalam S R, Maddala S and Krishna Prasad M H M 2012 Quality assessment of pixel level image fusion using fuzzy logic. Int. J. Soft Comput. 3(1): 11-23

[6] Kavitha S and Thyagharajan K K 2017 Efficient DWT-based fusion techniques using genetic algorithm for optimal parameter estimation. J. Soft Comput. 21(12): 3307-3316

[7] James A P and Dasarathy B V 2014 Medical image fusion: a survey of the state-of-the-art. Inf. Fus. 19: 4-19

[8] Barra V and Boire J V 2001 A general framework for the fusion of anatomical and functional medical images. Neurolmage 13(3): 410-424

[9] Khalegi B, Khamis A, Karray F O, et al 2013 Multisensor data fusion: a review of the state-of-the-art. Inf. Fus. 14(1): $28-44$

[10] Rajkumar S and Kavitha S 2010 Redundancy discrete wavelet transform and contourlet transform for multimodality medical image fusion with quantitative analysis. In: Proceedings of the 3rd IEEE International Conference on Emerging Trends in Engineering and Technology, pp. 134-139

[11] Shah P, Merchant S N and Desai U B 2013 Multifocus and multispectral image fusion based on pixel significance using multiresolution decomposition. Signal Image Video Process. 7(1): 95-109

[12] Bhateja V, Patel H, Krishn A, Sahu A and Lay-Ekuakille A 2015 Multimodal medical image sensor fusion framework using cascade of wavelet and contourlet transform domains. IEEE Sens. J. 15(12) 6783-6790

[13] Yang Y, Park D S, Huang S and Rao N 2010 Medical image fusion via an effective wavelet-based approach. EURASIP J. Adv. Signal Process. 2010: 579341

[14] Bhavana V and Krishnappa H K 2015 Multi-modality medical image fusion using discrete wavelet transform. In: Proceedings of 4th international conference on eco-friendly computing and communication systems, vol. 70, pp. 625-631

[15] Koley S, Galande A, Kelkar B, Sadhu A K, Sarkar D and Chakraborty C 2016 Multispectral MRI image fusion for enhanced visualization of meningioma brain tumors and 
edema using contourlet transform and fuzzy statistics. $J$. Med. Biol. Eng. 36(4): 470-484

[16] Srivastava R, Prakash O and Khare A 2016 Local energybased multimodal medical image fusion in curvelet domain. IET Comput. Vis. 10(6): 513-527

[17] Vijayarajan R 2015 Discrete wavelet transform based principal component averaging fusion for medical images. Int. J. Electron. Commun. 69(6): 896-902

[18] Lu H, Zhang L and Serikawa S 2012 Maximum local energy: an effective approach for multisensor image fusion in beyond wavelet transform domain. Comput. Math. Appl. 64(5): 996-1003

[19] Xu X, Wang Y and Chen S 2016 Medical image fusion using discrete fractional wavelet transform. Biomed. Signal Process. Control 27: 103-111

[20] De A, Kumar S K, Gunasekaran A and Tiwari M K 2017 Sustainable maritime inventory routing problem with time window constraints. Eng. Appl. Artif. Intell. 61: 77-95

[21] De A, Mamanduru V K R, Gunasekaran A, Subramanian N and Tiwari M K 2016 Composite particle algorithm for sustainable integrated dynamic ship routing and scheduling optimization. Comput. Ind. Eng. 96: 201-215

[22] Maiyar L M and Thakkar J J 2017 A combined tactical and operational deterministic food grain transportation model: particle swarm based optimization approach. Comput. Ind. Eng. 110: 30-42
[23] Irshad H, Kamran M, Siddiqui A B and Hussain A 2009 Image fusion using computational intelligence: a survey. In: Proceedings of the Second International Conference on Environmental and Computer Science, pp. 128-132

[24] Gonzalez R C and Woods R E 2009 Digital image processing. India: Pearson Education

[25] Choi M 2006 A new intensity-hue-saturation fusion approach to image fusion with a tradeoff parameter. IEEE Trans. Geosci. Remote Sens. 44(6): 1672-1682

[26] Nikolov S, Hill P, Bull D and Canagarajah N 2001 Wavelets for image fusion. In: Wavelets in signal and image analysis, vol. 19, pp. 213-241 (chapter)

[27] Mirjalili S and Lewis A 2016 The whale optimization algorithm. Adv. Eng. Softw. 95: 51-67

[28] Chander S, Vijaya P and Dhyani P 2016 Fractional Lion Algorithm - an optimization algorithm for data clustering. $J$. Comput. Sci. 12(7): 323-340

[29] Chander S, Vijaya P and Dhyani P 2016 MKF-firefly: hybridization of firefly and multiple kernel-based fuzzy C-means algorithm. Int. J. Adv. Res. Comput. Commun. Eng. 5(7): 213-216

[30] Gong Y J, et al 2016 Genetic learning particle swarm optimization. IEEE Trans. Cybernet. 46(10): 2277-2290

[31] https://www.smir.ch/BRATS/Start2015 